Supplementary Information for

\title{
Three-Dimensional Printable, Extremely Soft, Stretchable, and Reversible Elastomers from Molecular Architecture-Directed Assembly
}

Shifeng Nian, ${ }^{1, \dagger}$ Jinchang Zhu, ${ }^{1, \dagger}$ Haozhe Zhang, ${ }^{2}$ Zihao Gong, ${ }^{1}$ Guillaume Freychet, ${ }^{3}$ Mikhail Zhernenkov, ${ }^{3}$ Baoxing Xu, ${ }^{2} \mathrm{Li}-H e n g$ Cai ${ }^{1,4,5, *}$

${ }^{1}$ Soft Biomatter Laboratory, Department of Materials Science and Engineering, University of Virginia, Charlottesville, VA 22904, USA

${ }^{2}$ Department of Aerospace and Mechanical Engineering, University of Virginia, Charlottesville, VA 22904, USA

${ }^{3}$ National Synchrotron Light Source-II, Brookhaven National Laboratory, Upton, NY 11973 , USA

${ }^{4}$ Department of Chemical Engineering, University of Virginia, Charlottesville, VA 22904, USA

${ }^{5}$ Department of Biomedical Engineering, University of Virginia, Charlottesville, VA 22904, USA

$\dagger$ These authors contribute equally

*Correspondence: L.-H. Cai, liheng.cai@virginia.edu

The PDF file includes:

SI Text

Figures S1-S8

Tables S1-S2

Movies S1-S8 


\section{SI Text}

${ }^{1} \mathbf{H}$ NMR characterization. We use ${ }^{1} \mathrm{H}$ NMR to determine the number of side chains per bottlebrush and the weight fraction of PBnMA. The former one is calculated based on the conversion of PDMS macromonomers to bottlebrush PDMS, which is measured by the NMR spectra of the raw reaction mixture, as documented in our previous publication. ${ }^{31}$ The weight fraction of PBnMA is determined based on the NMR spectra of purified triblock copolymers. For example, in Fig. S1b area a, $A_{\mathrm{BnMA}}$, corresponds to the two $\mathrm{H}$ on the methylene group of BnMA repeating unit. Area $\mathrm{b}, A_{\mathrm{PDMS}}$, corresponds to four $\mathrm{H}$ on the two carbon atoms connected with the silicon atom. Therefore, the degree of polymerization of PBnMA is $n_{\mathrm{BnMA}}=$ $n_{\mathrm{PDMS}} \times\left(A_{\mathrm{BnMA}} / 2\right) /\left(A_{\mathrm{PDMS}} / 4\right)=218$, in which $A_{\mathrm{BnMA}}=3.9, A_{\mathrm{PDMS}}=4.0$, and the number of side chains per bbPDMS is $n_{\text {PDMS }}=112$. The weight fraction of PBnMA is given by: $f=$ $\left(n_{\mathrm{BnMA}} \times m_{\mathrm{BnMA}}\right) /\left(n_{\mathrm{BnMA}} \times m_{\mathrm{BnMA}}+n_{\mathrm{PDMS}} \times m_{\mathrm{PDMS}}\right)=0.064$, in which the mass of BnMA monomer $m_{\mathrm{BnMA}}=176.2 \mathrm{~g} / \mathrm{mol}$, and that of a PDMS macromonomer $m_{\text {PDMS }}=5000 \mathrm{~g} / \mathrm{mol}$.

Gel permeation chromatography. GPC measurements are performed using TOSOH EcoSEC HLC-8320GPC system with two TOSOH Bioscience TSKgel GMH $\mathrm{HR}_{\mathrm{H}} \mathrm{M} 5 \mu \mathrm{m}$ columns in series and a refractive index detector at $40^{\circ} \mathrm{C}$. HPLC grade THF is used as the eluent with a flow rate of $1 \mathrm{~mL} / \mathrm{min}$. The samples are dissolved in THF with a concentration around $3 \mathrm{mg} / \mathrm{mL}$. The GPC traces of all bbPDMS polymers and the corresponding LBBL polymers are shown in Fig. S2a and Fig. S2b, respectively. The MW and PDI of all samples are summarized in Table 1.

Molecular weight distribution of polymers. To further quantify the distribution of MW, we perform modality analysis for the LBBL triblock copolymers. The retention profile of sample $\mathrm{S}_{500}$ is well-described by a bimodal distribution, one minor peak with a shorter retention time at $t_{s}=$ $11.11 \mathrm{~min}$ and the other major peak with a longer retention time at $t_{l}=12.06 \mathrm{~min}$, as shown in Fig. S2c. Since the area under a monomodal distribution denotes the total mass of the polymer, the weight fraction of the larger MW component is about $8.7 \%$.

To convert the weight fraction to number fraction, one needs to determine the relation between MW ratio and the retention time ratio. For GPC, the elution volume, or time $t$, is linearly proportional to the logarithmic of MW. Therefore, the MW of a polymer is: $M=a \exp \left(-t / t_{0}\right)$, where parameters $a$ and $t_{0}$ are to be determined by column calibration. Because there are no GPC standards for LBBL polymers, we use other two LBBL polymer samples $\mathrm{S}_{250}$ and $\mathrm{S}_{1500}$ with negligible shoulder peaks as the standards for calibration. The MW of middle block for these two LBBL polymers are about $250 \mathrm{~kg} / \mathrm{mol}$ and $1500 \mathrm{~kg} / \mathrm{mol}$, respectively. The average retention time is $12.61 \mathrm{~min}$ for $\mathrm{S}_{250}$, and that for $\mathrm{S}_{1500}$ is $11.36 \mathrm{~min}$. Considering the MW ratio is about 6 , it gives $t_{0} \approx 42 \mathrm{sec}$. Therefore, for the bimodal distribution of sample $\mathrm{S}_{500}$, the MW ratio between the two peaks is: $M_{t s} / M_{t l}=\exp \left[-\left(t_{s}-t_{l}\right) / t_{0}\right] \approx 4$. This suggests that the average MW of the minor component is about four times of the major one in the triblock copolymer. Considering that the mass fraction of the minor component is about $8.7 \%$, the corresponding number fraction, $2.5 \%$, is 
very small. Thus, we conclude that the non-monomodal distribution of LBBL polymers has negligible effects on both microstructure and macroscopic mechanical properties.

GISAXS results and discussion. Consistent with the random sphere microstructure, all samples exhibit a relatively broad scattering peak, as shown in Fig. S3b. The peak position, $q$, corresponds to a characteristic length of $2 \pi / q$. We calculate the average distance, $d$, between the centers of two neighboring spherical domains based on the average wavenumber of the primary peak location, and the error based on the peak width, as listed in Table 1. Interestingly, among all samples only $\mathrm{S}_{500}$ exhibits a secondary peak at a wavenumber slightly larger than the twice of the primary peak, suggesting a long-range ordering of the self-assembled microstructure. Considering that peak is relatively broad, the microstructure likely is body center cubic (BCC), the possible peak locations of which are $\sqrt{2}, \sqrt{3}, \sqrt{4}, \ldots$ relative to the primary peak. Further understanding the self-assembly of linear-bottlebrush-linear polymers will be the subject of future study. 

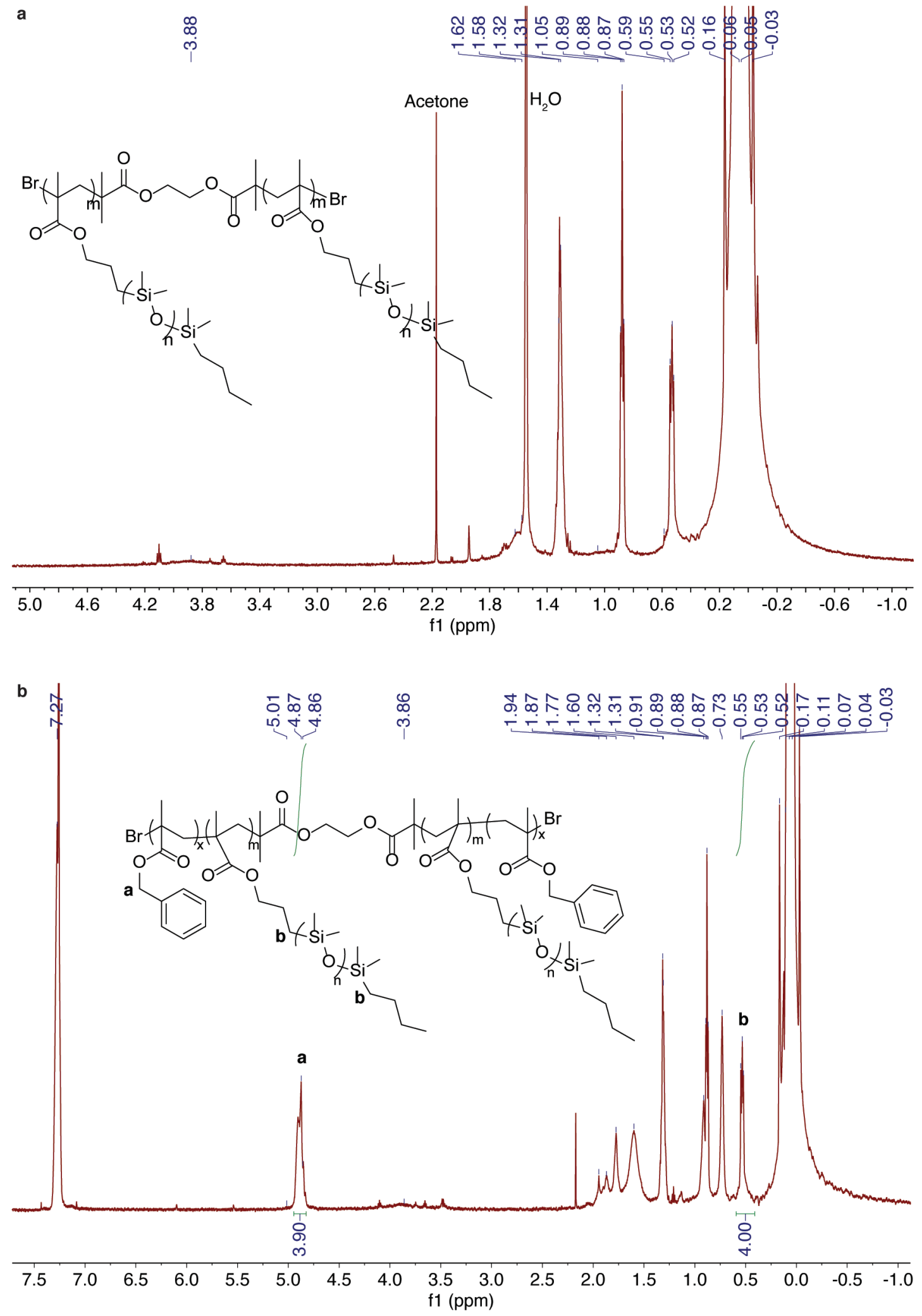
Fig. S1. ${ }^{1} \mathrm{H}$ NMR spectra of bbPDMS and LBBL polymers. (a) ${ }^{1} \mathrm{H}$ NMR spectrum (600 MHz, $\mathrm{CDCl}_{3}$ ) of a bottlebrush PDMS with the molecular weight of $560 \mathrm{k} \mathrm{g} / \mathrm{mol}$. (b) ${ }^{1} \mathrm{H} \mathrm{NMR}$ spectrum $\left(600 \mathrm{MHz}, \mathrm{CDCl}_{3}\right)$ of a PBnMA-bbPDMS-PBnMA LBBL triblock copolymer with the middle block of $560 \mathrm{~kg} / \mathrm{mol}$ and the weight fraction of PBnMA $6.4 \%$. 

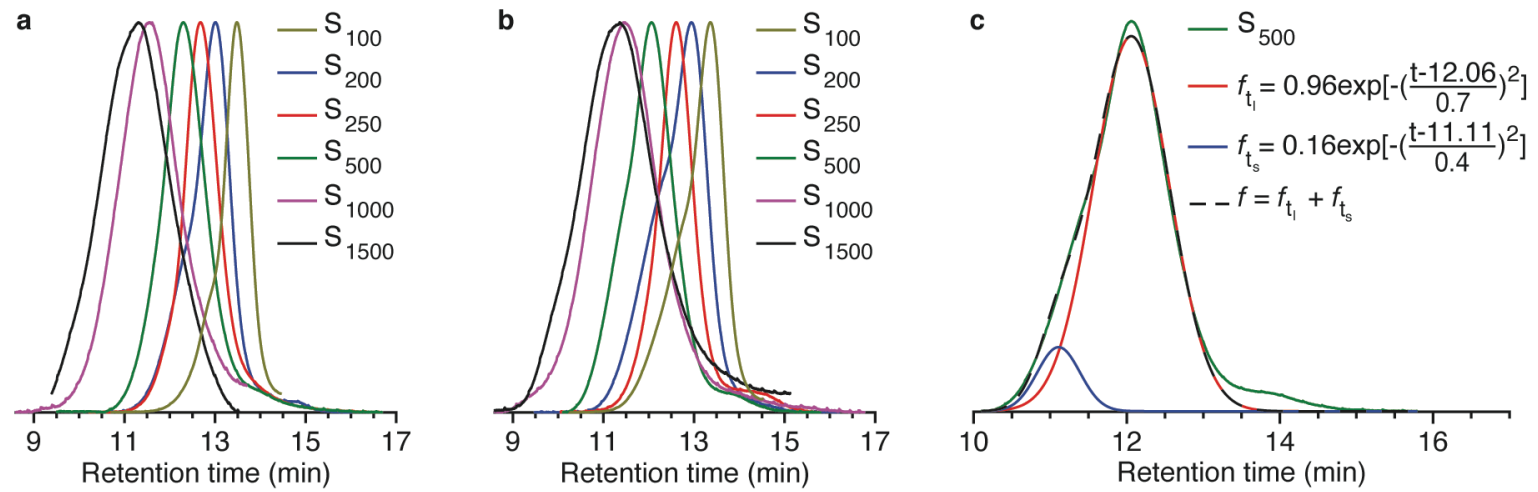

Fig. S2. Gel permeation chromatography (GPC) of all bbPDMS and LBBL polymers. (a) GPC trace of all bbPDMS polymers. (b) GPC trace of all LBBL triblock copolymers. (c) Modality analysis of sample $\mathrm{S}_{500}$ reveals a bimodal distribution. The larger component (blue line) has a weight fraction of $8.7 \%$, equivalent to a number fraction of $2.5 \%$. 

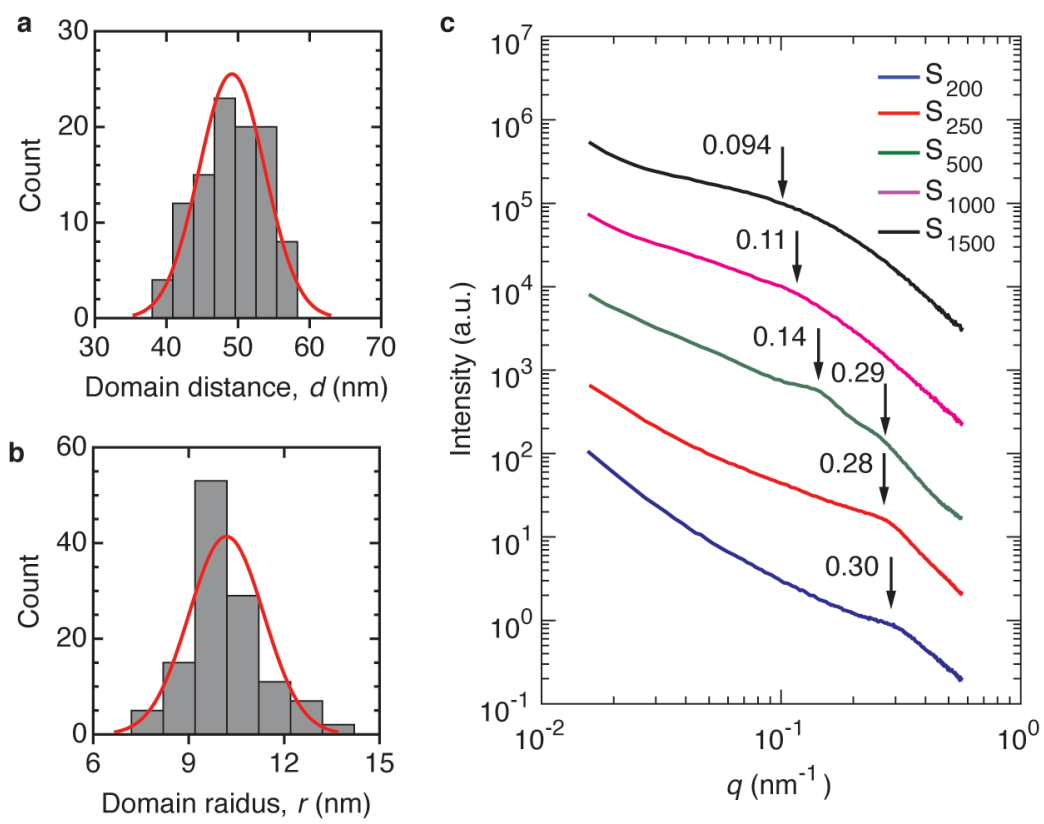

Fig. S3. Microstructure of ultrasoft elastomers. (a, b) Distributions of (a) the domain distance $d$ and (b) domain radius $r$ based on TEM images of sample $\mathrm{S}_{500}$ (Fig. 2b); $d=49.2 \pm 4.7 \mathrm{~nm}$, and $r=10.2 \pm 1.2 \mathrm{~nm}$. (c) Scattering intensity vs wavenumber $q$ for elastomers assembled by LBBL polymers of different molecular weight. 

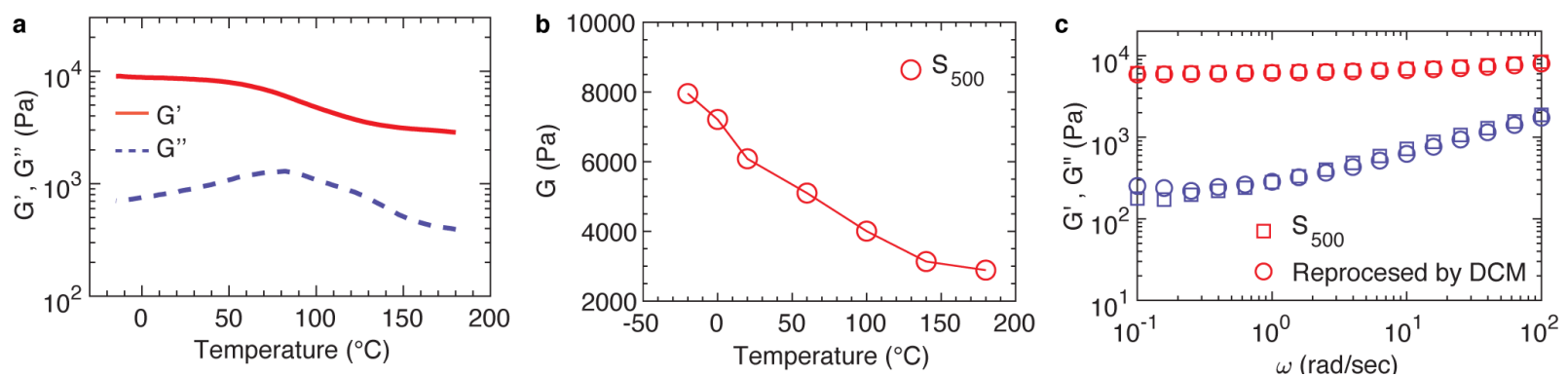

Fig. S4. Ultrasoft elastomers are thermostable yet solvent reprocessable. (a) Dependence of storage and loss moduli on temperature at a fixed strain of $5 \%$ and an oscillatory frequency of 1 $\mathrm{rad} / \mathrm{sec}$. (b) The equilibrium shear modulus is taken as the value of $G^{\prime}$ at the lowest oscillatory shear frequency $0.1 \mathrm{rad} / \mathrm{sec}$ (Fig. 2d). (c) Storage (red symbols, $G^{\prime}$ ) and loss (blue symbols, $G^{\prime \prime}$ ) moduli of sample $\mathrm{S}_{500}$ measured at a fixed strain of $0.5 \%$ at the temperature of $20^{\circ} \mathrm{C}$ before and after solvent reprocessing using dichloromethane (DCM). 


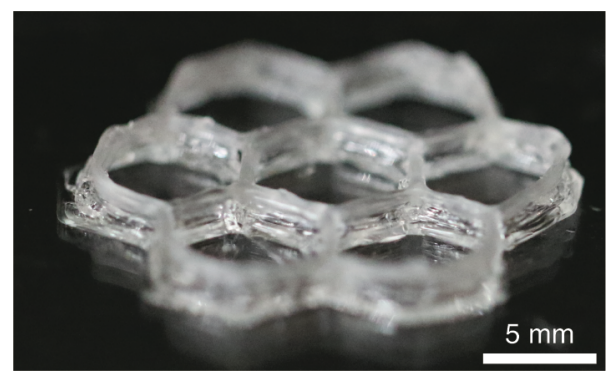

Fig. S5. A 3D printed honeycomb structure with a stack thickness of $2 \mathrm{~mm}$. 

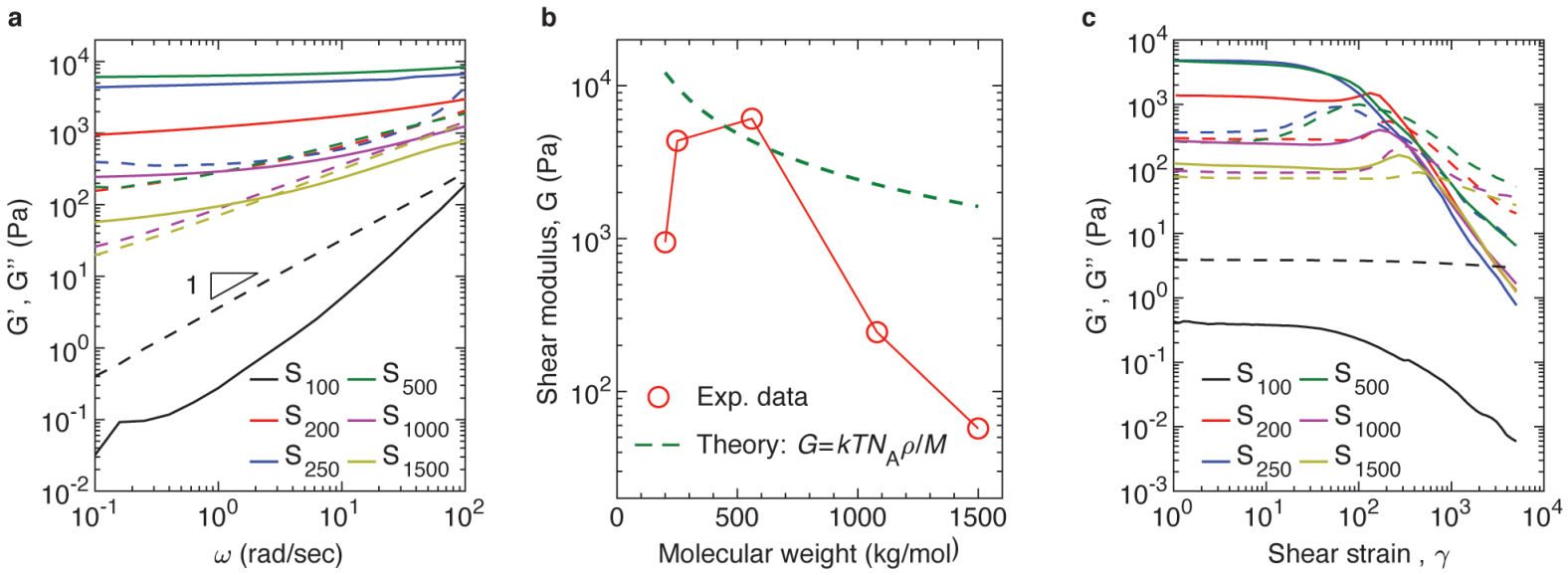

Fig. S6. Dependence of stiffness and extensibility on the molecular weight of LBBL polymers. (a) All samples are elastomers except for $\mathrm{S}_{100}$, which has a molecular weight of $100 \mathrm{kDa}$ and is liquid like. This is because the MW of the end linear blocks $3000 \mathrm{~g} / \mathrm{mol}$ is too small to form hard glassy domains. Solid lines, storage moduli $G$ '; dashed lines, loss moduli $G^{\prime}$ '. Measurements are performed at $0.5 \%$ strain and $20^{\circ} \mathrm{C}$. (b) Dependence of shear modulus (red circle) on the molecular weight of the bottlebrush middle block. Dashed line: Theoretical prediction for the shear modulus of an unentangled polymer network: $G=k T \rho N_{A} / M$, in which $N_{A}$ is Avogadro number, $\rho$ is polymer density, and $M$ is the molecular weight of the network strand. In the self-assembled network, the crosslinks have a finite volume. In the calculation, therefore, instead of using the mass of the middle block only, we use the total mass of a triblock copolymer, which dictates the volume occupied by each network strand. Nevertheless, this correction is negligible because the weight fraction of the end blocks is very small. (c) Large amplitude oscillatory shear measurements are performed at $1 \mathrm{rad} / \mathrm{sec}$ for all samples. The shear fracture strain increases when the elastomers become softer. 


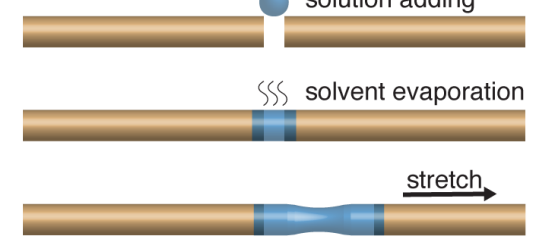

b

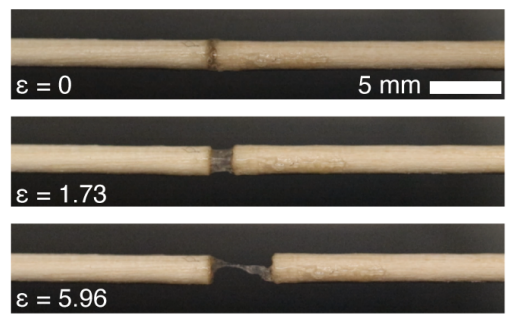

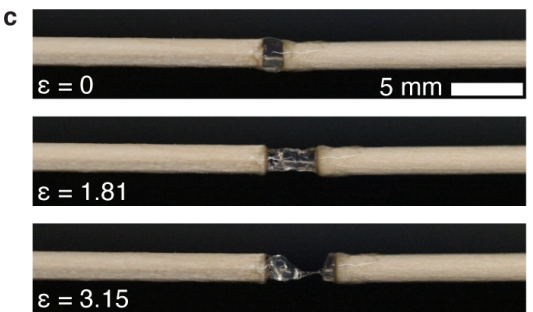

Fig. S7. Elongation tests for ultrasoft elastomers. (a) Illustration of experiment setup. $(b, c)$ Optical images of samples $S_{1500}$ (b) and $S_{1000}$ (c) under various extents of elongation at the rate of 0.01/sec (Movies S6\&7). 


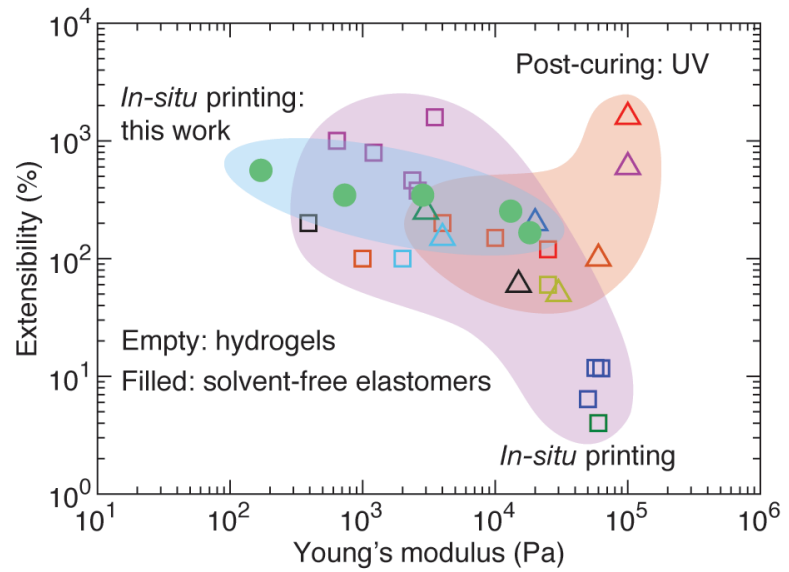

Fig. S8. Comparison of extensibility and stiffness between ultrasoft elastomers and existing 3D printable hydrogels. Filled circles are ultrasoft reversible elastomers. Empty symbols are existing 3D printable hydrogels listed in Table S2. 
Table S1. List of data points for Fig. 4B.

\begin{tabular}{|c|c|c|c|c|c|}
\hline Printing method & $\begin{array}{l}\text { Symbol } \\
\text { shape }\end{array}$ & $\begin{array}{c}\text { Symbol } \\
\text { color }\end{array}$ & $\begin{array}{l}\text { Young's } \\
\text { modulus } \\
(\mathrm{kPa})\end{array}$ & $\begin{array}{c}\text { Extensibility } \\
(\%)\end{array}$ & $\begin{array}{c}\text { Reference } \\
\text { number }\end{array}$ \\
\hline \multirow{17}{*}{$\begin{array}{l}\text { Thermoplastic } \\
\text { elastomers: in } \\
\text { situ printing + } \\
\text { post treatment }\end{array}$} & \multirow{17}{*}{ Square } & Black & 80000 & 60 & 1 \\
\hline & & \multirow{3}{*}{ Red } & 25000 & 860 & \multirow{3}{*}{2} \\
\hline & & & 25000 & 640 & \\
\hline & & & 25000 & 1030 & \\
\hline & & Blue & 2500 & 1600 & 3 \\
\hline & & Green & 11000 & 522 & 4 \\
\hline & & \multirow{2}{*}{ Yellow } & 2300 & 1150 & \multirow{2}{*}{5} \\
\hline & & & 2300 & 240 & \\
\hline & & \multirow{5}{*}{ Purple } & 85000 & 314 & \multirow{5}{*}{6} \\
\hline & & & 40000 & 260 & \\
\hline & & & 25000 & 230 & \\
\hline & & & 25000 & 170 & \\
\hline & & & 15000 & 140 & \\
\hline & & \multirow{4}{*}{ Orange } & 670 & 180 & \multirow{4}{*}{7} \\
\hline & & & 450 & 240 & \\
\hline & & & 220 & 280 & \\
\hline & & & 100 & 400 & \\
\hline \multirow{18}{*}{$\begin{array}{l}\text { Post-curing: } \\
\text { temperature }\end{array}$} & \multirow{18}{*}{ Circle } & Black & 125 & 900 & 8,9 \\
\hline & & \multirow{3}{*}{ Red } & 200 & 1000 & \multirow{3}{*}{3} \\
\hline & & & 350 & 930 & \\
\hline & & & 800 & 850 & \\
\hline & & \multirow{5}{*}{ Blue } & 3600 & 150 & \multirow{5}{*}{10} \\
\hline & & & 500 & 550 & \\
\hline & & & 400 & 480 & \\
\hline & & & 800 & 400 & \\
\hline & & & 150 & 2000 & \\
\hline & & \multirow{3}{*}{ Green } & 400 & 528 & \multirow{3}{*}{11} \\
\hline & & & 3610 & 362 & \\
\hline & & & 11510 & 77 & \\
\hline & & \multirow{6}{*}{ Yellow } & 1500 & 250 & \multirow{6}{*}{12} \\
\hline & & & 2000 & 190 & \\
\hline & & & 13000 & 130 & \\
\hline & & & 15000 & 110 & \\
\hline & & & 20000 & 80 & \\
\hline & & & 22000 & 50 & \\
\hline
\end{tabular}




\begin{tabular}{|l|c|c|c|c|c|}
\hline \multirow{2}{*}{} & \multirow{3}{*}{ Purple } & 13000 & 50 & \multirow{2}{*}{13} \\
\cline { 3 - 4 } & & 8000 & 60 & \multirow{2}{*}{} \\
\cline { 3 - 4 } & & 3000 & 60 & \\
\hline
\end{tabular}

Table S1. List of data points for Fig. 4B (continue).

\begin{tabular}{|c|c|c|c|c|c|}
\hline Printing method & $\begin{array}{l}\text { Symbol } \\
\text { shape }\end{array}$ & $\begin{array}{l}\text { Symbol } \\
\text { color }\end{array}$ & $\begin{array}{l}\text { Young's } \\
\text { modulus } \\
(\mathrm{kPa})\end{array}$ & $\begin{array}{c}\text { Extensibility } \\
(\%)\end{array}$ & $\begin{array}{c}\text { Reference } \\
\text { number }\end{array}$ \\
\hline \multirow{18}{*}{ Post-curing: UV } & \multirow{18}{*}{ Triangle } & \multirow{4}{*}{ Black } & 100 & 100 & \multirow{4}{*}{14} \\
\hline & & & 150 & 128 & \\
\hline & & & 300 & 140 & \\
\hline & & & 250 & 150 & \\
\hline & & \multirow{7}{*}{ Red } & 7500 & 1100 & \multirow{7}{*}{15} \\
\hline & & & 7000 & 1000 & \\
\hline & & & 4500 & 980 & \\
\hline & & & 2900 & 880 & \\
\hline & & & 1000 & 500 & \\
\hline & & & 900 & 400 & \\
\hline & & & 800 & 280 & \\
\hline & & \multirow{2}{*}{ Blue } & 1000 & 280 & \multirow{2}{*}{16} \\
\hline & & & 500 & 320 & \\
\hline & & \multirow{3}{*}{ Green } & 130 & 150 & \multirow{3}{*}{17} \\
\hline & & & 1000 & 267 & \\
\hline & & & 3400 & 922 & \\
\hline & & Yellow & 740 & 86 & 18 \\
\hline & & Purple & 7500 & 570 & 19 \\
\hline $\begin{array}{c}\text { Bottlebrush- } \\
\text { based elastomer }\end{array}$ & Diamond & Red & 32 & 410 & 20 \\
\hline
\end{tabular}


Table S2. List of data points for Fig. S8.

\begin{tabular}{|c|c|c|c|c|c|}
\hline Printing method & $\begin{array}{l}\text { Symbol } \\
\text { shape }\end{array}$ & $\begin{array}{l}\text { Symbol } \\
\text { color }\end{array}$ & $\begin{array}{c}\text { Young's } \\
\text { modulus (Pa) }\end{array}$ & $\begin{array}{c}\text { Extensibility } \\
(\%)\end{array}$ & $\begin{array}{l}\text { Reference } \\
\text { number }\end{array}$ \\
\hline \multirow{16}{*}{ In-situ printing } & \multirow{16}{*}{ Square } & Black & 390 & 200 & 21 \\
\hline & & \multirow{3}{*}{ Red } & 25000 & 120 & \multirow{3}{*}{22} \\
\hline & & & 10000 & 150 & \\
\hline & & & 4000 & 200 & \\
\hline & & \multirow{3}{*}{ Blue } & 50000 & 6.4 & \multirow{3}{*}{23} \\
\hline & & & 63000 & 11.7 & \\
\hline & & & 57000 & 11.8 & \\
\hline & & Green & 60000 & 4 & 24 \\
\hline & & Yellow & 25000 & 60 & 25,26 \\
\hline & & \multirow{5}{*}{ Purple } & 640 & 1000 & \multirow{5}{*}{27} \\
\hline & & & 1210 & 794 & \\
\hline & & & 2370 & 461 & \\
\hline & & & 3500 & 1587 & \\
\hline & & & 2600 & 378 & \\
\hline & & Cyan & 2000 & 100 & 28 \\
\hline & & Orange & 1000 & 100 & 26,29 \\
\hline \multirow{9}{*}{ Post-curing: UV } & \multirow{9}{*}{ Triangle } & Black & 15000 & 60 & 30 \\
\hline & & Red & 100000 & 1600 & 31 \\
\hline & & Blue & 20000 & 200 & 26,32 \\
\hline & & Green & 3000 & 250 & 22,26 \\
\hline & & Yellow & 30000 & 50 & 33,34 \\
\hline & & Purple & 100000 & 600 & 35 \\
\hline & & Cyan & 4000 & 150 & 34,36 \\
\hline & & Orange & 60000 & 100 & 26,29 \\
\hline & & Grey & 7000 & 1300 & 37 \\
\hline
\end{tabular}




\section{Reference}

(1) Vasquez, M.; Cross, J.; Hopkinson, N.; Haworth, B. Developing New Laser Sintering Materials for Snowboarding Applications. Procedia Eng. 2012, 34, 325-330. https://doi.org/10.1016/j.proeng.2012.04.056.

(2) Mo, J.; Kang, J.; Shin, S.; Jegal, J.; Gil, H.; Choy, S.; Hakkarainen, M.; Park, J.; Oh, D. X.; Yeon, S. Biobased Thermoplastic Elastomer with Seamless 3D-Printability and Superior Mechanical Properties Empowered by in-Situ Polymerization in the Presence of Nanocellulose. Compos. Sci. Technol. 2020, 185, 107885. https://doi.org/10.1016/j.compscitech.2019.107885.

(3) Sun, H.; Han, Z.; Willenbacher, N. Ultrastretchable Conductive Elastomers with a Low Percolation Threshold for Printed Soft Electronics. ACS Appl. Mater. Interfaces 2019, 11 (41), 38092-38102. https://doi.org/10.1021/acsami.9b11071.

(4) Kumar, N.; Jain, P. K.; Tandon, P.; Pandey, P. M. The Effect of Process Parameters on Tensile Behavior of 3D Printed Flexible Parts of Ethylene Vinyl Acetate (EVA). J. Manuf. Process. 2018, 35, 317-326. https://doi.org/10.1016/j.jmapro.2018.08.013.

(5) Valentine, A. D.; Busbee, T. A.; Boley, J. W.; Raney, J. R.; Chortos, A.; Kotikian, A.; Berrigan, J. D.; Durstock, M. F.; Lewis, J. A. Hybrid 3D Printing of Soft Electronics. Adv. Mater. 2017, 29 (40), 1703817. https://doi.org/10.1002/adma.201703817.

(6) Hu, X.; Kang, H.; Li, Y.; Geng, Y.; Wang, R.; Zhang, L. Preparation, Morphology and Superior Performances of Biobased Thermoplastic Elastomer by in Situ Dynamical Vulcanization for 3D-Printed Materials. Polymer. 2017, 108, 11-20. https://doi.org/10.1016/j.polymer.2016.11.045.

(7) Wallin, T. J.; Simonsen, L. E.; Pan, W.; Wang, K.; Giannelis, E.; Shepherd, R. F.; Mengüç, Y. 3D Printable Tough Silicone Double Networks. Nat. Commun. 2020, 11 (1), 1-10. https://doi.org/10.1038/s41467-020-17816-y.

(8) Muth, J. T.; Vogt, D. M.; Truby, R. L.; Kolesky, D. B.; Wood, R. J.; Lewis, J. A. Embedded 3D Printing of Strain Sensors within Highly Stretchable Elastomers. Adv. Mater. 2014, 26 (36), 6307-6312. https://doi.org/10.1002/adma.201400334.

(9) Park, Y.; Majidi, C.; Kramer, R.; Phillipe, B.; Wood, R. J. Hyperelastic Pressure Sensing with a Liquid-Embedded Elastomer. J. Micromechanics Microengineering 2010, 20 (12), 125029. https://doi.org/10.1088/0960-1317/20/12/125029.

(10) Zhou, L.; Gao, Q.; Fu, J.; Chen, Q.; Zhu, J.; Sun, Y. Multimaterial 3D Printing of Highly Stretchable Silicone Elastomers. ACS Appl. Mater. Interfaces 2019, 11 (26), 23573-23583. https://doi.org/10.1021/acsami.9b04873. 
(11) Durban, M. M.; Lenhardt, J. M.; Wu, A. S.; Iv, W. S.; Bryson, T. M.; Perez-perez, L.; Nguyen, D. T.; Gammon, S.; Smay, J. E.; Duoss, E. B.; et al. Custom 3D Printable Silicones with Tunable Stiffness. Macromol. Rapid Commun. 2018, 39 (4), 1700563. https://doi.org/10.1002/marc.201700563.

(12) Chortos, A.; Hajiesmaili, E.; Morales, J.; Clarke, D. R.; Lewis, J. A. 3D Printing of Interdigitated Dielectric Elastomer Actuators. Adv. Funct. Mater. 2020, 30 (1), 1907375. https://doi.org/10.1002/adfm.201907375.

(13) Chen, K.; Zhang, L.; Kuang, X.; Li, V.; Lei, M.; Kang, G.; Wang, Z. L.; Qi, H. J. Dynamic Photomask-Assisted Direct Ink Writing Multimaterial for Multilevel Triboelectric Nanogenerator. Adv. Funct. Mater. 2019, $29 \quad$ (33), 1903568. https://doi.org/10.1002/adfm.201903568.

(14) Xiang, H.; Wang, X.; Ou, Z.; Lin, G.; Yin, J.; Liu, Z. Progress in Organic Coatings UVCurable, 3D Printable and Biocompatible Silicone Elastomers. Prog. Org. Coatings 2019, 137 (100), 105372. https://doi.org/10.1016/j.porgcoat.2019.105372.

(15) Patel, D. K.; Sakhaei, A. H.; Layani, M.; Zhang, B.; Ge, Q.; Magdassi, S. Highly Stretchable and UV Curable Elastomers for Digital Light Processing Based 3D Printing. Adv. Mater. 2017, 29 (15), 1606000. https://doi.org/10.1002/adma.201606000.

(16) Davidson, E. C.; Kotikian, A.; Li, S.; Aizenberg, J.; Lewis, J. A. 3D Printable and Reconfigurable Liquid Crystal Elastomers with Light-Induced Shape Memory via Dynamic Bond Exchange. Adv. Mater. 2020, $32 \quad$ (1), 1905682. https://doi.org/10.1002/adma.201905682.

(17) Schaffner, M.; Faber, J. A.; Pianegonda, L.; Rühs, P. A.; Coulter, F.; Studart, A. R. 3D Printing of Robotic Soft Actuators with Programmable Bioinspired Architectures. Nat. Commun. 2018, 9 (1), 1-9. https://doi.org/10.1038/s41467-018-03216-w.

(18) Yeh, Y. C.; Highley, C. B.; Ouyang, L.; Burdick, J. A. 3D Printing of Photocurable Poly(Glycerol Sebacate) Elastomers. Biofabrication 2016, 8 (4), 045004. https://doi.org/10.1088/1758-5090/8/4/045004.

(19) Kuang, X.; Chen, K.; Dunn, C. K.; Wu, J.; Li, V. C. F.; Qi, H. J. 3D Printing of Highly Stretchable, Shape-Memory, and Self-Healing Elastomer toward Novel 4D Printing. ACS Appl. Mater. Interfaces 2018, 10 (8), 7381-7388. https://doi.org/10.1021/acsami.7b18265.

(20) Xie, R.; Mukherjee, S.; Levi, A. E.; Reynolds, V. G.; Wang, H.; Chabinyc, M. L.; Bates, C. M. Room Temperature 3D Printing of Super-Soft and Solvent-Free Elastomers. Sci. Adv. 2020, 6 (46), eabc6900. https://doi.org/10.1126/sciadv.abc6900.

(21) Shin, M.; Song, K. H.; Burrell, J. C.; Cullen, D. K.; Burdick, J. A. Injectable and Conductive Granular Hydrogels for 3D Printing and Electroactive Tissue Support. Adv. Sci. 2019, 6 
(20), 1901229. https://doi.org/10.1002/advs.201901229.

(22) Wang, L. L.; Highley, C. B.; Yeh, Y. C.; Galarraga, J. H.; Uman, S.; Burdick, J. A. ThreeDimensional Extrusion Bioprinting of Single- and Double-Network Hydrogels Containing Dynamic Covalent Crosslinks. J. Biomed. Mater. Res. - Part A 2018, 106 (4), 865-875. https://doi.org/10.1002/jbm.a.36323.

(23) Li, L.; Zhang, P.; Zhang, Z.; Lin, Q.; Wu, Y.; Cheng, A.; Lin, Y.; Thompson, C. M.; Smaldone, R. A.; Ke, C. Hierarchical Co-Assembly Enhanced Direct Ink Writing. Angew. Chemie 2018, 130 (18), 5199-5203. https://doi.org/10.1002/ange.201800593.

(24) Wu, W.; Deconinck, A.; Lewis, J. A. Omnidirectional Printing of 3D Microvascular Networks. Adv. Mater. 2011, 23 (24), 178-183. https://doi.org/10.1002/adma.201004625.

(25) Ouyang, L.; Highley, C. B.; Sun, W.; Burdick, J. A. A Generalizable Strategy for the 3D Bioprinting of Hydrogels from Nonviscous Photo-Crosslinkable Inks. Adv. Mater. 2017, 29 (8), 1604983. https://doi.org/10.1002/adma.201604983.

(26) Rodell, C. B.; Dusaj, N. N.; Highley, C. B.; Burdick, J. A. Injectable and Cytocompatible Tough Double-Network Hydrogels through Tandem Supramolecular and Covalent Crosslinking. $\quad A d v$. Mater. 2016, $28 \quad$ (38), 8419-8424. https://doi.org/10.1002/adma.201602268.

(27) Rutz, A. L.; Hyland, K. E.; Jakus, A. E.; Burghardt, W. R.; Shah, R. N. A Multimaterial Bioink Method for 3D Printing Tunable, Cell-Compatible Hydrogels. Adv. Mater. 2015, 27 (9), 1607-1614. https://doi.org/10.1002/adma.201405076.

(28) Highley, C. B.; Song, K. H.; Daly, A. C.; Burdick, J. A. Jammed Microgel Inks for 3D Printing Applications. Adv. Mater. 2019, $6 \quad$ (1), 1801076. https://doi.org/10.1002/advs.201801076.

(29) Highley, C. B.; Rodell, C. B.; Burdick, J. A. Direct 3D Printing of Shear-Thinning Hydrogels into Self-Healing Hydrogels. Adv. Mater. 2015, 27 (34), 5075-5079. https://doi.org/10.1002/adma.201501234.

(30) Schaffner, M.; Rühs, P. A.; Coulter, F.; Kilcher, S.; Studart, A. R. 3D Printing of Bacteria into Functional Complex Materials. Sci. Adv. 2017, 3 (12), eaao6804. https://doi.org/10.1126/sciadv.aao6804.

(31) Yang, H.; Li, C.; Yang, M.; Pan, Y.; Yin, Q.; Tang, J.; Qi, H. J.; Suo, Z. Printing Hydrogels and Elastomers in Arbitrary Sequence with Strong Adhesion. Adv. Funct. Mater. 2019, 29 (27), 1901721. https://doi.org/10.1002/adfm.201901721.

(32) Ouyang, L.; Highley, C. B.; Rodell, C. B.; Sun, W.; Burdick, J. A. 3D Printing of ShearThinning Hyaluronic Acid Hydrogels with Secondary Cross-Linking. ACS Biomater. Sci. Eng. 2016, 2 (10), 1743-1751. https://doi.org/10.1021/acsbiomaterials.6b00158. 
(33) Barry, R. A.; Shepherd, R. F.; Hanson, J. N.; Nuzzo, R. G.; Wiltzius, P.; Lewis, J. A. DirectWrite Assembly of 3D Hydrogel Scaffolds for Guided Cell Growth. Adv. Mater. 2009, 21 (23), 2407-2410. https://doi.org/10.1002/adma.200803702.

(34) Calvet, D.; Wong, J. Y.; Giasson, S. Rheological Monitoring of Polyacrylamide Gelation: Importance of Cross-Link Density and Temperature. Macromolecules 2004, 37 (20), 77627771. https://doi.org/10.1021/ma049072r.

(35) Hong, S.; Sycks, D.; Chan, H. F.; Lin, S.; Lopez, G. P.; Guilak, F.; Leong, K. W.; Zhao, X. 3D Printing of Highly Stretchable and Tough Hydrogels into Complex, Cellularized Structures. $\quad A d v . \quad$ Mater. $2015, \quad 27 \quad$ (27), 4035-4040. https://doi.org/10.1002/adma.201501099.

(36) Tian, K.; Bae, J.; Bakarich, S. E.; Yang, C.; Gately, R. D.; Spinks, G. M.; Suo, Z.; Vlassak, J. J. 3D Printing of Transparent and Conductive Heterogeneous Hydrogel - Elastomer Systems. Adv. Mater. 2017, 29 (10), 1604827. https://doi.org/10.1002/adma.201604827.

(37) Zhang, B.; Li, S.; Hingorani, H.; Serjouei, A.; Larush, L.; Pawar, A. A.; Goh, W. H.; Sakhaei, A. H.; Hashimoto, M.; Kowsari, K.; et al. Highly Stretchable Hydrogels for UV Curing Based High-Resolution Multimaterial 3D Printing. J. Mater. Chem. B 2018, 6 (20), 32463253. https://doi.org/10.1039/c8tb00673c. 